\title{
Original Research Paper Oncology \\ MANAGING CHILDHOOD CANCER AMIDST COVID-19 PANDEMIC IN A PUBLIC HEALTH FACILITY IN INDIA
}

\section{Nita \\ Radhakrishnan*}

Ravi Shankar
MD, FNB, Assistant Professor, Department of Pediatric Hematology Oncology, Super Speciality Pediatric Hospital and Post Graduate Teaching Institute, Noida, Delhi NCR, India *Corresponding Author

MD, Fellow, Department of Pediatric Hematology Oncology, Super Speciality Pediatric Hospital and Post Graduate Teaching Institute, Noida, Delhi NCR, India

MBBS, Junior Resident, Department of Pediatric Hematology Oncology, Super Speciality Pediatric Hospital and Post Graduate Teaching Institute, Noida, Delhi NCR, India

Medical Social Worker, Department of Pediatric Hematology Oncology, Super Speciality Pediatric Hospital and Post Graduate Teaching Institute, Noida, Delhi NCR, India COVID-19 infection and the logistic support available. Even in the same country, often different strategies have to be developed to ensure patients do not stop treatment for want of facilities. In this qualitative paper, we describe the difficulties faced since the onset of COVID-19 pandemic and how solutions emerged during the course of time. We conclude that the usual hand-holding needed for families with childhood cancer need to be augmented during these tough times to ensure no child is denied treatment for cancer.

KEYWORDS : COVID-19, Cancer, Children

\section{INTRODUCTION}

Since the onset of the COVID-19 pandemic in December 2019, there have been concerns raised regarding the care of children with cancer during this global crisis. The concerns have been mostly related to susceptibility of children to the disease and potential risk of increased mortality if infected with COVID-19 (1,2). There are a multitude of indirect concerns of this pandemic with childhood cancer. These include nonavailability of beds for cancer treatment as many government centers treating childhood cancer were converted to COVID hospitals and difficulty in elective surgeries for biopsies and definitive care. Most patients faced difficulty in traveling to treatment centers due to lockdown, absence of public transport and financial constraints due to economic slowdown. Difficult in access to medication like chemotherapy and antibiotics, blood products and supportive care treatment made matters worse. In addition to all, there is an extreme fear of COVID-19 which prompts patient families to stay indoors and refuse essential treatment which is often life threatening. In this article, we describe the difficulties faced at a government center in North India and the solutions that emerged during the course of 3 months. We anticipate similar constraints in other low and middle income countries and expect that this discussion will help us generate regional solutions that are easily adaptable.

\section{METHODS}

We describe the series of constraints faced between 15 March 2020 to 15 Aug 2020 (5 months) while managing children aged $0-18$ years with cancer at our tertiary care center in North India. Our centre is a pediatric tertiary care teaching hospital located in North India in the National Capital Region. We cater to patients from Uttar Pradesh, Delhi and other adjoining states such as Uttarakhand, Bihar and Punjab. We diagnose and manage 60-70 new patients annually and have a follow up of more than 350 children with cancer.

Since the beginning of COVID-19 related planning, the Government had issued guidelines for essential treatment services such as chemotherapy, blood transfusion and emergency care to continue (3). In view of this, modifications were made for chemotherapy (described later) and patients were being managed with teleconsultation, shared care with pediatricians and admissions when absolutely necessary. However, since the last week of April 2020, when the centre was being converted as a COVID hospital, there was risk of complete cessation of services. With the kind permission of the government and hospital administration, a day-care was permitted to continue essential chemotherapy and blood transfusions to ensure no child died of cancer due to lack of access to care. The issues that emerged during this period were prospectively recorded and repeated sessions of discussion with other faculty, social workers, nongovernmental organisations (NGO) supporting cancer care, blood donation groups helped generate solutions to these issues.

\section{RESULTS}

During the study period of 5 months, there were 1124 visits of childhood cancer in our day-care clinic. This constituted visits from 83 paediatric cancer patients of which 33 were new diagnoses during this period. The newly diagnosed patients included acute lymphoblastic leukemia (ALL) (36\%), acute myeloid leukemia (AML) (13.8\%), Hodgkin and non-Hodgkin lymphoma $(11 \%)$ and solid tumours $(33 \%)$ such as Wilms tumour, Neuroblastoma, Ewing sarcoma, Synovial sarcoma, Hepatoblastoma and Langerhan Cell Histiocytosis. 3 patients with brain tumour were referred to another centre for surgery and one child with bilateral Wilms tumour diagnosed during this period was lost to follow up.

\section{Diagnosis of cancer}

1. Most cancer evaluation including bone marrow evaluation, tissue biopsies and cytology has remained the same. Open surgical biopsies and difficult biopsies often needing interventional radiology was not possible and we had to rely on tumour markers (hepatoblastoma, neuroblastoma) whenever possible.

2. Flow cytometry, molecular evaluation and minimal residual disease monitoring continued as before. 
Treatment strategy adopted during this period

1. All patients who had completed treatment for any cancer were not called to the hospital during this period unless for an emergency.

2. Most patients on maintenance chemotherapy for ALL and acute promyelocytic leukemia (APML) and chronic myeloid leukemia were not called to the hospital or regular follow up. Teleconsultations were offered and blood count based modification of oral chemotherapy drugs were advised. Medicines wherever not available were provided either with NGO support.

3. Induction chemotherapy for six new ALL patients and continuation of chemotherapy for rest were given as per schedule. Since there was no provision for overnight admission, patients were retained in the day care between 8am to 8pm and called again the next day for continuation of antibiotics/ supportive care. Standard 3 drug induction was followed for induction. No modification of ALL chemotherapy was done.

4. Two AML patients received first induction during this period. Currently, they are in the 3-4 week of therapy and are clinically stable. One child was transferred from another centre where she completed first induction and she was managed subsequently here.

5. No treatment modification was made for most lymphomas and solid tumours other than increasing the time interval between cycles thus making it less dose intensive. This would reduce the risk of sepsis and transfusions. In case of treatment regimens with cisplatin and ifosphamide, intravenous hydration was given for 12 hours with emphasis on adequate oral hydration at night under antiemetic cover of Ondansetron and Domperidone.

6. In case of 2 children with Burkitt Lymphoma on LMB protocol with High dose methotrexate, due to logistic difficulties of administering methotrexate and folinic acid, $\mathrm{R}-\mathrm{CHOP}$ was given instead.

7. No elective surgery was possible during this period. Hence surgery was delayed for 2 children Ewing sarcoma after ensuring good treatment response and chemotherapy was continued.

Healthcare staff

1. We had shortage of staff nurses as most of the department staff who were trained in cancer care and handling of central lines were posted to the COVID unit.

2. We ensured continuity of care of doctors (team of 1 consultant, 1 senior resident and 1 junior resident) throughout the last 5 months.

3. All nurses who worked in the department for one week was given quarantine and were tested for COVID-19 in case of any symptoms. This ensured better compliance from the nursing staff. One main nurse was continued daily for part-time and she took care of the central line dressings and major chemotherapy for the day.

4. Ancillary staff such as nutritionist, social workers, counsellor, teacher etc. worked 1-2 days a week during the initial period of 3 months.

COVID and cancer

During this period, 2 children were diagnosed with COVID-19 infection. One child a 15-year old girl, was diagnosed as suspected leukemia at another facility and referred for further treatment. On admission, she was found to be hypoxic and hence was tested for COVID-19 which was positive. She was managed in COVID ward and on evaluation she was diagnosed as ALL which was subsequently managed. She continues to be in remission and is well. The second case a 2year-old boy had a retroperitoneal mass (suspected Wilms tumour) who developed fever and rash at home; subsequently he was tested and found to be COVID-19 positive. He recovered uneventfully and was referred to another centre for biopsy.
COVID prevention

All standard precautions for COVID prevention including PPE with $\mathrm{N} 95$ masks for all healthcare staff and screening at the entrance of the day-care clinic with temperature check and history of fever/cough was implemented from the beginning. Handwashing was promoted among patients and attendants. Nasopharyngeal sample for rapid antigen for chemotherapy was done in those who had suspected symptoms. Uniform screening for COVID-19 was not feasible in our scenario due to lack of healthcare staff and turnaround time. Also, attendants with fever, cough and other respiratory complaints were turned back and only one attendant was allowed for the patient.

\section{Adverse events from treatment}

1. No undue side effect was noted due to any of the treatment modification.

2. Febrile neutropenia was encountered as usual and responded with antimicrobial support and GCSF.

3. Mortality was encountered in two of the newly diagnosed patients. Both these children were brought from their homes with sepsis and shock and could not be salvaged despite all efforts including ICU admission at another centre.

\section{Fear and anxiety of COVID}

1. Fear and anxiety was probably the biggest challenge we encountered. Most patients in the initial 2 months expressed fear in stepping out of their homes. 5 children with ALL on maintenance had stopped treatment temporarily. Subsequently with appropriate counselling this was overcome.

2. Fear of COVID was encountered in nursing staff, social workers and parent support team members. Appropriate supply of personal protective equipment and adherence to strict regimen of handwashing, masks and social distancing helped them overcome this fear. In the initial few months, most staff were asked to report to work only l2 days a week. This also helped with social distancing and boosted their morale.

Travel during COVID-19 lockdown

1. Most patients expressed difficulty to travel to the centre. For those who requited continuous care, provision was made to stay in nearby home-away-from-home and continue care.

2. Ambulance service was provided by CanKids NGO to ferry patients from their homes to hospital and vice versa

\section{Financial support}

1. Cancer chemotherapy, supportive care and hospital expenses including investigations are usually provided free to patients through various government schemes. Even for those who were not on government schemes, help was received from NGOS to support their treatment.

2. Application for government schemes were significantly delayed as many patients were not able to apply for 'income certificate' and other mandatory government documents due to lockdown. Once applied however, funds were sanctioned in time and this helped the treatment immensely.

3. Reimbursement for those who were not on schemes was arranged for blood component therapy and travel with the help of an NGO, Jiv Daya Foundation.

\section{Blood complement transfusion}

1. Blood component transfusion was the most difficult aspect of treatment of cancer. Due to lock down, donation camps have not been possible since the last 5 months. The help of blood donor groups was sought to motivate donors to come to hospital for donation. All standard precautions were taken in the blood bank by the Department of 
Transfusion Medicine for preventing COVID-19 infection in donors.

2. Standard guidelines for blood component therapy (haemoglobin $<8 \mathrm{gm} / \mathrm{dl}$ and platelet count $<20,000 / \mu \mathrm{L}$ ) was followed as much as possible. At times however due to non-availability of blood products, transfusion was delayed by 1-2 days. No patient however had an adverse event related to transfusion during this period.

\section{Shared care}

1. During the initial days of COVID-19 related lockdown, although cancer care was considered essential service, many patients experienced difficulty in reaching the hospital. In this situation, help of paediatricians in the locality of the patient was sought to administer antibiotics and GCSF in patients with febrile neutropenia.

2. A network of social workers and NGOs was available to discuss difficulties faced by each patient and to arrive at $a$ customised solution. Often parents themselves helped each other by providing blood donors and travel support.

\section{DISCUSSION}

The pandemic created by SARS-COV-2 has been the most unprecedented one. Such unexpected challenges often force us to find unexpected solutions. Even though cancer care was included as an essential service, patients still found it difficult to reach the treatment centre (3). Hence alternative methods to treat had to be devised to reduce patient visits to the hospital to a minimum. However, despite this, a significant number of patients had to been seen and managed, failing which they had a high risk of disease recurrence. Thus all efforts were focussed on those children who needed support not just for travel but through the entire process of treatment. With the current strategy, we have been able to provide care for all with no patient absconding treatment due to COVID-19 related constraints.

There have been similar guidance and experience sharing from other centres in India which describe decision based on accessibility to reach hospital. Although our strategy was also similar, we were also constrained in terms of financial difficulties of patients and in-patient beds (4). Thus, institutional strategies need to be customised to their set of constraints and guidelines provide only an overview in this situation. A similar description from Italy mention routine RT PCR for all patients at triage and for all fever patients (5). These experiences have been helpful in charting our own strategies $(6,7)$.

Finally, in childhood cancer, it takes a village to treat. This adage has gained more importance during this pandemic as we could not have managed without the help of all the stake holders. With the risk of cancer patients developing COVID-19 and subsequent morbidity for patient, family and healthcare workers, the situation for non-COVID diseases such as cancer has been grim (8). We wish to acknowledge that the support from the state government and administration has been priceless. Their support has helped these patients to continue treatment, as majority of our patients would have stopped treatment otherwise. The role of NGOs in arranging for travel, medicines, provision of masks and sanitizers for patients and financial support has helped us immensely. And finally the willpower of the healthcare staff to tide over this crisis has been the crux of this effort.

\section{REFERENCES}

1. Zimmermann $\mathrm{P}$, Curtis N. Coronavirus Infections in Children Including COVID-19: An Overview of the Epidemiology, Clinical Features, Diagnosis, Treatment and Prevention Options in Children. Pediatr Infect Dis J. 2020 May; 39(5):355-368.

2. Terenziani M, Massimino M, Biassoni V, Casanova M, Chiaravalli S, Ferrari A et al. SARS-CoV-2 disease and children under treatment for cancer. Pediatr Blood Cancer. 2020 May 6: e28346.

3. Enabling Delivery of Essential Health Services during the COVID 19
Outbreak: Guidance note. Retrieved from https://www.mohfw.gov.in/pdf /EssentialservicesduringCOVID19updated0411201.pdf

4. Garg V, Bakhshi S, Gupta G, Pushpam D. Managing Pediatric Cancer Patients in COVID19 Pandemic. IndianJ Pediatr. 2020;87(7):558.

5. Molica M, Mazzone C, Cordone I, Pasquale A, Niscola P, de Fabritiis P. SARS$\mathrm{CoV}-2$ infection anxieties and general population restrictions delay diagnosis and treatment of acute haematological malignancies. Br J Haematol. 2020;190(1):e5-e8.

6. Sainati L, Biffi A. How we deal with the COVID-19 epidemic in an Italian paediatric onco-haematology clinic located in a region with a high density of cases. Br J Haematol. 2020; 189(4):640-642.

7. Gulia A, Arora RS, Panda PK et al. Adapting Management of Sarcomas in COVID-19: An Evidence-Based Review Indian J Orthop. 2020 May 30 : 1-13.

8. Dai M, Liu D, Liu M, et al. Patients with Cancer Appear More Vulnerable to SARS-CoV-2: A Multicenter Study during the COVID-19 Outbreak. Cancer Discov. 2020;10(6):783-791. 